\title{
Altered Blood Vessel Responses in the Eye and Finger in Coronary Artery Disease
}

\author{
Rebekka Heitmar, ${ }^{1}$ Robert P. Cubbidge, ${ }^{1}$ Gregory Y. H. Lip, ${ }^{1,2}$ Doina Gherghel, ${ }^{1}$ \\ and Andrew D. Blann ${ }^{2}$
}

Purpose. Cardiac function, such as heart rate variability, is abnormal in coronary artery disease, but its relation with the function of ocular and nail-fold blood vessels is unknown. The hypothesis was that there is abnormal retinal and peripheral microvascular endothelial function compared with large blood vessel and cardiac function. Twenty-four patients with coronary artery disease (CAD) and 30 healthy, age- and sex-matched control subjects were enrolled in the study.

Methods. Peripheral microcirculatory function was measured with continuous retinal vessel diameter assessment and nailfold capillaroscopy. Systemic vascular function was evaluated by 24-hour blood pressure, arterial stiffness, low (LF)- and high (HF)-frequency heart rate variability, ECG monitoring, and the plasma markers von Willebrand factor (vWf) and soluble $\mathrm{E}$ selectin.

REsults. Peripheral nail-fold capillary $(P=0.009)$ and retinal vessel (average baseline corrected flicker response [BFR]; $P=$ $0.034)$ responses and reaction time in response to flicker ( $P=$ 0.016 ) were significantly different in patients compared with controls. Furthermore, patients demonstrated higher arterial stiffness $(P=0.005), \mathrm{LF}$ and $\mathrm{HF}$ heart rate variability $(P=$ $0.004, P=0.006)$, and vWf level $(P=0.044)$, but there was no difference in soluble E selectin level $(P=0.278)$. In the CAD patients, LF and HF heart rate variability both correlated with average BFR $(r=0.58, P=0.004 ; r=-0.6, P=0.003$, respectively). There was no such relationship in the healthy controls.

Conclusions. Microcirculatory abnormalities of the retina and nail-fold vessels are present in CAD. The two indices of heart rate variability correlated with an index of ocular vessel responses. The latter may be a surrogate marker of abnormal heart rate variability in CAD. (Invest Ophthalmol Vis Sci. 2011; 52:6199-6205) DOI:10.1167/iovs.10-6628

B lood vessel tone is regulated both locally (for example, by the production of endothelin and nitric oxide by the endothelium) and centrally (by the effects of the autonomic nervous system on medial smooth muscle cells). ${ }^{1,2}$ Commonly used measures of vascular function and reactivity include systolic and diastolic blood pressure (SBP/DBP) at the brachial artery and heart rate variability (HRV), as measured by electro-

From the ${ }^{1}$ Aston University, School of Life and Health Sciences, Birmingham, United Kingdom; and the ${ }^{2}$ University of Birmingham Centre for Cardiovascular Sciences, City Hospital, Birmingham, United Kingdom.

Submitted for publication September 23, 2010; revised November 12, 2010, and January 6 and 31, 2011; accepted February 25, 2011.

Disclosure: R. Heitmar, None; R.P. Cubbidge, None; G.Y.H. Lip, None; D. Gherghel, None; A.D. Blann, None

Corresponding author: Rebekka Heitmar, Aston University, School of Life and Health Sciences, Aston Triangle, B4 7ET, Birmingham, UK r.heitmar1@aston.ac.uk. cardiogram (ECG). Both indices are known to vary over a 24-hour period because of an alteration in the balance in sympathetic and parasympathetic activity. ${ }^{3,4}$

The major pathophysiological drivers of atherosclerosis, manifesting in the clinical setting as coronary artery disease (CAD), include thrombosis and hypertension, and both of these are related to endothelial dysfunction. ${ }^{5}$ Subjects with CAD also display altered diurnal blood pressure cycling, reduced heart rate variability, and increased arterial stiffness. ${ }^{6-8}$ While these abnormalities are reflective of large artery disease, there is also evidence of disturbance in small arteries and arterioles, down to the level of the peripheral vascular beds (i.e., the microcirculation), ${ }^{9,10}$ an example of which is retinal disease, as is often present in diabetic and hypertensive retinopathy. ${ }^{11,12}$ However, it is as yet unclear whether microcirculatory changes are contributors to, or sequelae of, the disease process.

Microcirculatory function can be assessed using techniques such as laser Doppler flowmetry, tissue oximetry, nail-fold capillaroscopy, and retinal vessel reactivity, ${ }^{13-16}$ and these may be surrogates of systemic vascular disease. ${ }^{17-19}$ Noninvasive retinal vessel analysis could be a useful tool, not only for early diagnosis of ocular disease but also to assist diagnosis and for monitoring of the progression of systemic vascular disease. ${ }^{18}$ Alterations of the ocular vessel wall and structure, both conjunctival and retinal, can also occur and is detected by fundus photography and conjunctival images. ${ }^{9}$ For example, ocular vessel dysfunction has been demonstrated in diabetic patients with CAD, as the spectral wave shape of the color Doppler measurements at the retrobulbar vessels level exhibits a flattening of the systolic phase. ${ }^{20}$

It is also known that large-artery stiffness is increased, not only in hypertensive and prehypertensive patients, but also in patients who have CAD ${ }^{21-23}$ although to date no research has assessed retinal vessel reactivity in patients with CAD. Furthermore, other evidence of endothelial perturbation in CAD comes from increased levels of the plasma markers von Willebrand factor (vWf) and soluble E selectin. ${ }^{24}$ Nonetheless, new technologies assessing the ocular and nail-fold circulation may determine functional abnormalities in the function of a vascular bed that reflect pathologies in major organs, such as the heart and the brain. ${ }^{25,26}$ As many largevessel vascular changes occur before the development of CAD, such microcirculatory changes may also be predictive of future systemic pathology.

We therefore hypothesized that subjects with established CAD have altered indices of peripheral microcirculatory disease (e.g., nail-fold capillaroscopy and ocular vessel abnormalities), when compared to healthy subjects, and that these correlate with systemic indices such as heart rate variability and blood pressure. We tested our hypothesis in a simple case-control study. 


\section{Materials AND Methods}

\section{Subjects}

We recruited 24 patients with established CAD, proven by coronary angiography, from a postmyocardial infarction cardiac rehabilitation unit. Abnormal blood vessel physiology in this group was confirmed by heart rate variability, large-artery stiffness, and the presence of the plasma markers vWf and soluble E selectin. Age-matched, healthy individuals were recruited from the patients' spouses and other volunteers. Exclusion criteria were age $<18$ years, connective tissue disease, cancer, stroke, diabetes mellitus (DM), hypertension (HT: SBP/DBP > $140 / 90 \mathrm{~mm} \mathrm{Hg}$ ), atrial fibrillation (AF), stroke, history of ocular disease (i.e., patients with a refractive error of more than $\pm 3 \mathrm{D}$ spherical equivalent and more than $\pm 1 \mathrm{D}$ cylindrical equivalent; required to address possible magnification/minification and so a cause of over- or underestimation of the retinal diameter measured), or a history of neurologic diseases associated with loss of visual function or any type of ocular surgery. Approval was obtained from the West Birmingham and Aston University ethics committees. Written informed consent was received from all individuals taking part in the study. The study was designed and conducted in accordance with the Declaration of Helsinki.

\section{Study Protocol}

A full history and physical examination were conducted, to ensure that the subjects were free of any disease, as outlined in the exclusion criteria. All subjects were instructed to refrain from consuming caffeinated products, chocolate, and alcohol and from smoking on the study day. In addition, they were asked to abstain from their usual medication 24 hours before the appointment, in an attempt to minimize the effects of medication and in accordance with previous studies and with practice in cardiovascular disease research. ${ }^{27}$ All subjects underwent the test procedure outlined below.

\section{Systemic Hemodynamic Parameters}

Ambulatory Blood Pressure Monitoring. Twenty-four hours of blood pressure and ECG data were collected from each patient and control (Cardiotens-01; Meditech Ltd., Budapest, Hungary). All subjects completed a diary giving information about any use of medication (dosage and time) and physical activities (e.g., walking or other rigorous exercise). Day and night periods were estimated based on true sleep and wake-up times for each individual participating in the study. SBPs and DBPs were used to obtain an ambulatory arterial stiffness index (AASI), calculated according to the formula: AASI $=1-$ slope of the regression fit of the DBP as a function of the SBP (using BP recordings of a 24-hour monitoring time). ${ }^{28,29}$ The closer the value of AASI to 1, the stiffer the arterial tree of a given subject.

Heart Rate Variability. HRV analysis assesses autonomic nervous system (ANS) function by estimating sympathetic and parasympathetic activity and can be analyzed in either time- or frequencydomain analyses of ECG recordings: The latter was used as it focuses on global variations. ${ }^{30}$ Recordings were Fourier transformed to obtain power spectra, and from the result, two frequency ranges were assessed: low frequency (LF, 0.04-0.15 Hz) and high frequency $(\mathrm{HF}$, 0.15-0.40 Hz) (Cardiovision 1.7.2 software; PMS Instruments, Ltd., Maidenhead, UK). LF is mainly a measure of sympathetic activity with a minor influence of parasympathetic activity, whereas HF represents solely parasympathetic activity. ${ }^{31,32}$ Therefore, an LF/HF ratio measures sympathovagal balance, an increase of this ratio indicating a predominance of the sympathetic versus parasympathetic nervous system activity. Values for LF, HF, and LF/HF ratios were obtained from the 24-hour ECG recordings and were extracted similarly to the BP recordings for daytime and nighttime and circadian changes. Circadian changes were calculated for each LF and HF and the LF/HF ratio of each participant, by using the equation: Circadian change HRV = active period HRV - passive period HRV.

\section{Ocular Circulation}

Intraocular Pressure. IOP was measured by contact tonometry and calculated as the mean of three consecutive readings after instillation of 1 drop of $0.4 \%$ benoxinate hydrochloride (Chauvin Pharmaceuticals Ltd., Kingston-on-Thames, UK, TonopenXL; Medtronic Solan, PMS Instruments, Maidenhead, UK). Data were discarded if the coefficient of variation (CV) exceeded 5\%.

Retrobulbar Blood Flow Velocities. The patient was positioned supine with the head on a pillow for the 15-minute acclimatization period necessary to reach stable BP. Color Doppler ultrasound was used to collect the following data: (1) Blood flow velocities were measured in the ophthalmic artery (OA), central retinal artery (CRA), and posterior ciliary arteries (PCAs; Acuson Sequoia; Siemens, Newbury, UK) ${ }^{33}$ Peak systolic velocity (PSV), and end diastolic velocities (EDVs) of each OA, CRA, and medial and temporal PCA were measured on the recorded spectral pulse wave. Resistive indices (RIs) were calculated according to RI $=(\mathrm{PSV}-\mathrm{EDV}) / \mathrm{PSV}$. (2) The spectral wave form of the blood flow velocities was measured in the OA and CRA. Based on the appearance of the spectral wave, subjects were grouped into normal or abnormal wave shape (i.e., a flattened systolic phase) for both the OA and CRA. ${ }^{20}$ Figure 1 illustrates a normal and an abnormal spectral wave shape: the former had a clear, sharp peak, and the latter had a far smaller, rounded peak.

Dynamic and Static Retinal Vessel Assessment. After full pupil dilation was obtained with $1 \%$ tropicamide (Chauvin Pharmaceuticals, Ltd.), digital fundus images and reactivity parameters of retinal blood arteries and veins were obtained (Retinal Vessel Analyzer [RVA]; Imedos Systems (UG), Jena, Germany). ${ }^{34}$ For static vessel analysis, black-and-white fundus images were obtained at a $30^{\circ}$ angle with the optic nerve head centered, with the inbuilt fundus camera (model 450F; Carl Zeiss Meditec, GmbH, Oberkochen, Germany). Arterial and venous diameters provided an arteriovenous ratio (AVR), central retinal artery equivalent (CRAE), and central retinal vein equivalent (CRVE) (Vesselmap software; Imedos Systems, UG). ${ }^{35,36}$ CRVE, CRAE, and AVR were calculated from arteries and veins that were located within a ring whose center was the optic nerve head and whose inner and outer margins were of one-half disc diameter (DD) and one full DD. These measurements (AVR, CRAE, and CRVE) are standard ophthalmic indices and are used to describe the physical structure of different retinal arteries and veins, such as luminal diameter.

Static imaging was followed by dynamic assessment, where retinal diameters were measured continuously at a sampling rate of $25 \mathrm{~Hz}$. Stimulation of retinal blood vessels was done by optoelectronic interruption of the green fundus illumination used by the RVA resulting in a flickering light provocation with a $12.5-\mathrm{Hz}$ frequency. ${ }^{37-39}$ After BP stabilization and image focusing, a vessel segment of the superior temporal retinal artery and vein (40 measurement units; $500 \mu \mathrm{m}$ in length, according to the Gullstand model eye) was chosen at a distance of 1.5 to 2 disc diameters away from the margins of the optic nerve head. The baseline diameters of both the artery and vein were recorded according to the standard RVA protocol ${ }^{39}$ for 50 seconds, followed by three cycles of 20-second flicker provocation, each with 80 seconds of recovery time. This resulted in a 350-second measuring period during which the fellow eye was occluded. From the diameter recordings, the values for maximum dilation (MD), maximum constric-

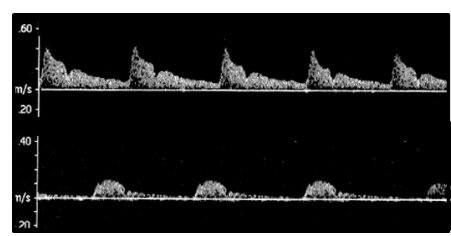

FigURE 1. Spectral wave shape recorded by color Doppler ultrasound. Spectral wave shape of the ophthalmic artery of (top) a healthy individual and (bottom) a subject with coronary artery disease. Vertical axis: blood flow velocity $(\mathrm{m} / \mathrm{s})$, horizontal axis: time. 


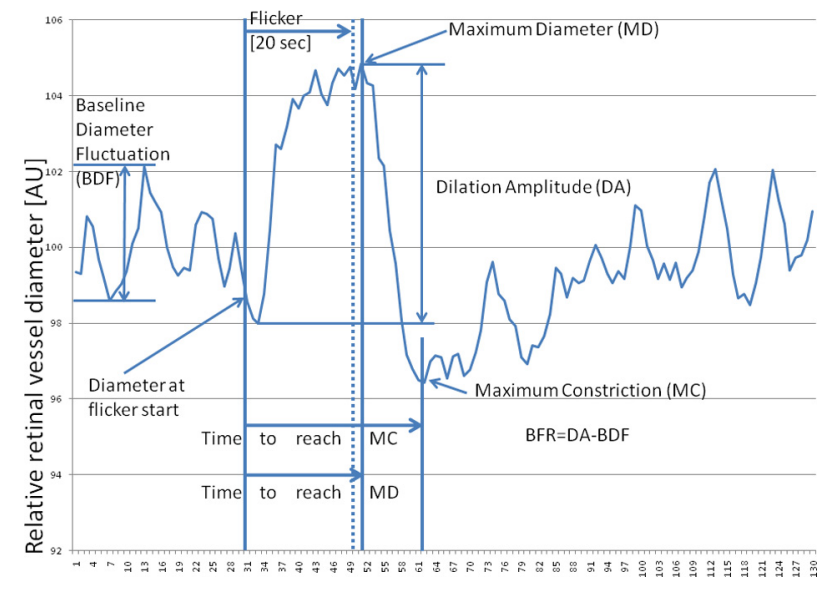

FIGURE 2. Changes in a retinal vessel as it is stimulated by flickering light. The horizontal axis is time (seconds). Left: BDF defines the fluctuation in the baseline diameter. Middle: an increase is shown in relative vessel diameter due to flickering light. This response provides the MD, MC, DA, and the time to reach MD and MC. Right: the responses of the vessel as it recovers.

tion (MC), and dilation amplitude (DA), arterial baseline corrected flicker response (BFR), and arterial and venous reaction time (RT) in response to flicker provocation were calculated ${ }^{40}$ (Fig. 2).

Reproducibility measurements of dynamic retinal parameters in a sample of 10 healthy controls showed good agreement between the paired measurements. Interassay $\mathrm{CV}$ for retinal arteries were $4 \%$ for baseline diameter, $7.3 \%$ for MD, $4.1 \%$ for MC, $3.7 \%$ for DA, 3.4\% for BFR, and $7.1 \%$ for arterial reaction time. Venous CVs showed similar results: $3.8 \%$ for baseline diameter, $4.6 \%$ for $\mathrm{MD}, 2.1 \%$ for $\mathrm{MC}, 4.6 \%$ for $\mathrm{DA}$, and $4.7 \%$ for venous reaction time. Intra-assay CVs and other technical data have been described in detail elsewhere. ${ }^{40}$

\section{Peripheral Circulation}

Capillaroscopy of the front row nail-fold capillaries was used to assess peripheral microcirculation. It has been used to evaluate peripheral vasospasm in patients with angina and other systemic vascular diseases, ${ }^{41-43}$ An infrared laser beam is directed at the nail fold with a fiber optic probe, and blood flow is determined from the measured frequency Doppler shift. (CAM1 Laser Doppler Capillary Anemometer; KK Technology, Devon, UK). Immediately before capillary flow was measured, both hands of each subject were immersed in warm water $\left(40^{\circ} \mathrm{C}\right.$ ) for 3 minutes to maximize blood flow and to therefore minimize any influence of seasonal temperature variations on flow variables. ${ }^{44-46}$ Data were collected according to the following procedure: (1) Baseline measurement. After image focusing (front row capillaries of the nail-fold area of the right-hand ring finger), a baseline measurement of at least 30 seconds was obtained by means of laser Doppler flowmetry to obtain baseline capillary flow (BCF). (2) Cold provocation with carbon dioxide. The nail-fold area was cooled immediately after baseline data acquisition by rapidly decompressing carbon dioxide for 60 seconds. Stream temperature was calibrated at $-15^{\circ} \mathrm{C}$ to cool the nail-fold area down to $+15^{\circ} \mathrm{C} .^{43}$ (3) Recovery time. This period was defined as the time needed (in seconds) for capillary flow to return to baseline values after cessation of cold provocation.

\section{Plasma Markers}

After the subjects fasted overnight and refrained from systemic medication, venous blood was collected into citric acid, and plasma was obtained after centrifugation at $1000 \mathrm{~g}$ for 20 minutes. Levels of von Willebrand factor (vWf) and soluble E selectin were measured by commercial enzyme-linked immunosorbent assay (ELISA; Dako-Cytomation, Cambs, UK, and R\&D Systems, Abingdon, UK). The ELISA's had intra- and interassay coefficients of variation $<5 \%$ and $<10 \%$, respectively.

\section{Power Calculation}

Our pilot data ${ }^{40}$ reported a significant difference in retinal vein diameter in 21 smokers (mean $\pm \mathrm{SD}, 161 \pm 6 \mu \mathrm{m}$ ) compared with 21 ageand sex-matched nonsmokers $(150 \pm 16 \mu \mathrm{m} ; P=0.018)$. We therefore based our present sample size on the hypothesis of a similar difference (i.e., two thirds of an SD) in the retinal vein of patients with CAD. For a $1-\beta$ of 0.9 and $\alpha<0.05$, a sample size of 48 is required. Accordingly, we recruited consecutive subjects to a sample size of at least 24 per group. We recruited more controls (to $n=30$ ) for added confidence. In addition, this sample size provides the power $(\alpha=0.05$, $1-\beta=0.8$ ) to detect a correlation coefficient $(r)$ of $0.5 .{ }^{47}$

\section{Statistical Analysis}

Continuous data were subjected to the Shapiro-Wilks test, to determine distribution. If normally distributed, they are presented as the mean with SD, with the groups compared by $t$-test. Data distributed nonnormally are presented as the median with interquartile range (IQR), analyzed by the Mann-Whitney U test. Categorical data were compared using the $\chi^{2}$ test. Serial data were analyzed by repeated-measures analysis of variance or Friedman's method. Data were correlated using Pearson's method (if normally distributed) or Spearman's method (if non-normally distributed). With multiple analyses, in simple intergroup testing (see Tables 2,3 ), we set a more stringent probability at $P<0.01$ for statistical significance. For data in Tables $4 \mathrm{~A}$ and $4 \mathrm{~B}$, the analyses were inherently more robust (repeated measures analysis of variance and Friedman's methods) and so we do not have to reduce the level of probability from $P<0.05$ (Statistica ver. 6.0; Statsoft, Tulsa, OK).

\section{Results}

Patient and controls were matched for age, sex, and blood pressure, but the patients had a higher body mass index than the controls (Table 1). The difference in proportions of men and women (23\% of controls are women, $12.5 \%$ of CAD patients are women) is not significant. We confirmed endothelial abnormalities in patients with plasma markers and with assessment of HRV and arterial stiffness. CAD patients had signifi-

TABLE 1. Clinical and Demographics

\begin{tabular}{lccc}
\hline \multicolumn{1}{c}{ Parameter } & $\begin{array}{c}\text { Controls } \\
(\boldsymbol{n}=\mathbf{3 0 )})\end{array}$ & $\begin{array}{c}\text { CAD } \\
(\boldsymbol{n}=\mathbf{2 4})\end{array}$ & $\boldsymbol{P}$ \\
\hline Sex, M/F & $23 / 7$ & $21 / 3$ & 0.309 \\
Age, y & $53(9)$ & $56(9)$ & 0.162 \\
BMI, kg/m ${ }^{2}$ & $26(3)$ & $28(4)$ & 0.023 \\
SBP, mm Hg & $120(12)$ & $121(13)$ & 0.938 \\
DBP, mm Hg & $75(9)$ & $73(12)$ & 0.883 \\
Previous ACS & - & 24 & \\
CABG & - & 9 & \\
PCTA + Stent & - & 6 & \\
No intervention & - & 3 & \\
Aspirin & - & 20 & \\
Statins & - & 21 & \\
ACEIs & - & 17 & \\
Clopidogrel & - & 10 & \\
$\mathrm{H}_{2}$ receptor antagonists & - & 3 & \\
Ca channel antagonists & - & 5 & \\
Selective $\beta 1$ blocker & - & 19 & \\
Other drugs (nicorandil, paroxetine, & - & 4 & \\
$\quad$ alendronate, candesartan) & & & \\
\hline
\end{tabular}

Data are presented as mean and standard deviation or as number of patients. CABG, coronary artery bypass graft; PCTA, percutaneous transluminal angioplasty; ACS, acute coronary syndrome; ACEIs, angiotensin-converting enzyme inhibitors. 
TABLE 2. Blood Pressure and Heart Rate Variability Parameters

\begin{tabular}{|c|c|c|c|}
\hline Parameter & Controls $(n=30)$ & Patients $(n=24)$ & $P$ (between Groups) \\
\hline \multicolumn{4}{|l|}{ 24-b Blood Pressure } \\
\hline SBP/DBP day, mm Hg & $125(11) / 76(8)$ & $120(15) / 73(9)$ & $0.381 / 0.369$ \\
\hline SBP/DBP night, $\mathrm{mm} \mathrm{Hg}$ & $110(10) / 63(7)$ & $106(14) / 62(9)$ & $0.311 / 0.531$ \\
\hline SBP/DBP $24 \mathrm{~h}, \mathrm{~mm} \mathrm{Hg}$ & $120(10) / 72(7)$ & $117(10) / 68(8)$ & $0.469 / 0.114$ \\
\hline AASI, AU & $0.24(0.09)$ & $0.35(0.15)$ & 0.005 \\
\hline \multicolumn{4}{|c|}{ Heart Rate Variability from 24-b ECG } \\
\hline LF day, NU & $75(8)$ & $70(10)$ & 0.067 \\
\hline LF night, NU & $67(13)$ & $73(10)$ & 0.092 \\
\hline Within group & $<0.001$ & 0.291 & \\
\hline HF day, NU & $23(7)$ & $26(8)$ & 0.205 \\
\hline HF night, NU & $31(12)$ & $25(9)$ & 0.047 \\
\hline Within group & $<0.001$ & 0.689 & \\
\hline LF/HF night, NU & $2.8(1.4$ to 3.6$)$ & $3.5(2.2$ to 4.5$)$ & 0.069 \\
\hline LF/HF day, NU & $3.8(2.8$ to 4.1$)$ & $3.2(2.1$ to 3.9$)$ & 0.114 \\
\hline Within group & 0.002 & 0.638 & \\
\hline Day/night LF, NU & $7.9(0.0$ to 13.0$)$ & $-2.9(-13.0$ to 6.0$)$ & 0.004 \\
\hline Day/night HF, NU & $-8.3(-14.0$ to -2.0$)$ & $1.0(-7.0$ to 9.0$)$ & 0.006 \\
\hline Day/night LF/HF, NU & $1.0(0.3$ to 1.6$)$ & $-0.3(-1.7$ to 0.9$)$ & 0.027 \\
\hline
\end{tabular}

Data presented as the mean (SD) or the mean (IQR), depending on distribution. AASI, Ambulatory Arterial Stiffness Index; NU, normalized units.

cantly increased vWF levels $(88 \pm 15 \mathrm{IU} / \mathrm{dL}$ vs. $82 \pm 8 \mathrm{IU} / \mathrm{dL}$; $P=0.044)$ but not soluble E selectin $(55.2 \pm 12.7 \mathrm{ng} / \mathrm{mL}$ vs. $51.0 \pm 13.0 \mathrm{ng} / \mathrm{mL} ; P=0.278$ ) compared with healthy controls. AASI was increased in CAD patients compared with the controls, even though there were no differences in day, night, or mean 24-hour BP (Table 2). HRV analysis using the 24-hour ECG recordings showed no differences in the day or night LF activity (reflecting sympathetic activity), or daytime HF activity (reflecting parasympathetic activity) between the groups. Nighttime HF activity was marginally lower in the patients. There was an (expected) decline LF activity in the healthy controls from day to night while HF activity increased, whereas in the patients, neither change was present. Day and night changes in LF, HF, and their ratio (a measure of sympathovagal balance) were altered in the patients compared with the controls. The controls had on average $7.8 \pm$ 1.1 hours of sleep, whereas the patients had $7.9 \pm 1.1$ hours $(P=0.604)$.

Table 3 shows ocular parameters. There were no differences in IOP, being $13 \pm 3 \mathrm{~mm} \mathrm{Hg}$ in the controls versus $14 \pm$ 4 in the patients $(P=0.694)$. The retrobulbar blood velocity of the OA, CRA, and CRV were comparable between the study groups, but CRVE was higher in the patients. The dynamic retinal vessel parameters are shown in Tables $4 \mathrm{~A}$ (arteries) and $4 \mathrm{~B}$ (veins). There were no significant differences in mean arterial and venous diameters in the patients and controls. In the arteries (Table 4A), continuous retinal diameter analysis showed comparable results for $\mathrm{MD}, \mathrm{MC}$, and DA between the study groups. Baseline corrected flicker response (BFR) showed an altered reaction pattern in the dilatory response of patients compared with controls. Furthermore, reaction time to flicker stimulation was, despite a similar baseline reaction time, significantly different between the groups, in that the patients needed progressively longer for each stimulation cycle, whereas the controls' arterial reaction time was comparable between cycles. All venous data were comparable between the groups (Table 4B).

Spectral wave shape analysis of the OA and CRA revealed no significant difference in the proportion of CAD patients with flattened systolic phase in the OA $(n=10$ abnormal wave shape, 14 normal) compared with the controls ( 6 abnormal, 24 normal; $P=0.083$ ). In the CRA, parallel data were 16 abnormal wave shapes and 8 normal wave shapes in the patients, compared with 7 abnormal and 23 normal wave shapes in the controls $(P=0.001)$. For illustration, Figure 1 shows the spectral wave shape of the OA in a healthy person and a patient with CAD.

\section{Finger Blood Flow and Reactivity}

Blood velocity in CAD patients needed considerably longer to recover after cold provocation than in the controls (CAD median, 35; IQR, 15-50 seconds; controls, 21; 15-25 seconds; $P=$ $0.009)$. However, there was no difference in nail-fold capillary blood velocity in the patients (BCF $0.18 \pm 0.06 \mathrm{~mm} / \mathrm{s}$ ) compared with the controls $0.23 \pm 0.11 \mathrm{~mm} / \mathrm{s}(P=0.064)$.

\section{Correlations}

We performed the Spearman correlation analyses to determine any possible relationships between indices of vascular function

TABLE 3. Ocular Parameters

\begin{tabular}{llll}
\hline & Controls & Patients \\
Parameter & $(n=30)$ & $(n=24)$ & $P$ \\
\hline
\end{tabular}

Retrobulbar Blood Flow as Measured with Color Doppler Ultrasound

$\begin{array}{cccc}\text { OA (PSV), mm/s } & 36(8) & 40(9) & 0.107 \\ \text { (EDV), mm/s } & 9(4) & 0.76(0.06) & 0.654 \\ \text { CRA (PSV), mm/s } & 11(3) & 12(3) & 0.969 \\ \text { (EDV), mm/s } & 3.4(2.5 \text { to } 4.1) & 3.6(2.2 \text { to } 4.7) & 0.611 \\ \text { (RI) } & 0.70(0.08) & 0.68(0.10) & 0.622 \\ \text { CRV (Max), mm/s } & 6.4(4.6 \text { to } 6.7) & 7.3(5.0 \text { to } 9.1) & 0.275 \\ \text { (Min), mm/s } & 4.2(2.9 \text { to } 4.5) & 4.8(3.3 \text { to } 5.3) & 0.302\end{array}$

Static Retinal Vessel Measures Obtained by Retinal Photography

$\begin{array}{lccc}\text { AVR, ratio } & 0.85(0.09) & 0.83(0.09) & 0.923 \\ \text { CRAE, AU } & 193(26) & 201(21) & 0.331 \\ \text { CRVE, AU } & 232(26) & 251(26) & 0.041\end{array}$

Data presented in mean and standard deviation or mean and inter quartile ranges depending on distribution. AU, arbitrary units. 
TABLE 4. Dynamic Retinal Vessel Diameters

\begin{tabular}{|c|c|c|c|}
\hline \multicolumn{4}{|c|}{ A. Arterial Response } \\
\hline Parameter & Controls $(n=30)$ & Patients $(n=24)$ & $\boldsymbol{P}$ \\
\hline Size artery, AU & $114(18)$ & $118(16)$ & 0.471 \\
\hline \multicolumn{4}{|l|}{$\mathrm{MD}, \%$} \\
\hline F1 & $5.8(2.9$ to 7.1$)$ & $5.9(3.0$ to 8.4$)$ & 0.499 \\
\hline F2 & $6.0(3.7$ to 8.0$)$ & $4.9(2.3$ to 6.5$)$ & \\
\hline F3 & $6.0(4.4$ to 6.1$)$ & $5.7(3.1$ to 6.9$)$ & \\
\hline Intergroup $P$ & 0.472 & 0.165 & \\
\hline \multicolumn{4}{|l|}{ MC, $\%$} \\
\hline F1 & $-2.5(-4.3$ to -0.6$)$ & $-2.6(-3.3$ to -1.4$)$ & 0.148 \\
\hline F2 & $-2.4(-3.5$ to -1.5$)$ & $-1.8(-2.6$ to -1.0$)$ & \\
\hline F3 & $-1.8(-2.9$ to -0.6$)$ & $-2.2(-3.3$ to -0.8$)$ & \\
\hline Intergroup $P$ & 0.060 & 0.229 & \\
\hline \multicolumn{4}{|l|}{ DA, $\%$} \\
\hline F1 & $8.4(4.4$ to 11.8$)$ & $8.2(4.9$ to 12.1$)$ & 0.305 \\
\hline $\mathrm{F} 2$ & $8.4(5.4$ to 9.8$)$ & $6.7(3.7$ to 9.6$)$ & \\
\hline F3 & $7.8(5.5$ to 9.7$)$ & $7.9(4.6$ to 10.6$)$ & \\
\hline Intergroup $P$ & 0.779 & 0.064 & \\
\hline \multicolumn{4}{|l|}{ BFR, \% } \\
\hline F1 & $4.3(1.6$ to 6.4$)$ & $3.7(1.0$ to 5.5$)$ & 0.607 \\
\hline F2 & $4.4(2.2$ to 6.0$)$ & $2.9(0.1$ to 4.7$)$ & 0.194 \\
\hline F3 & $4.0(2.1$ to 5.1$)$ & $3.3(0.4$ to 5.8$)$ & 0.430 \\
\hline Intergroup $P$ & 0.564 & 0.034 & \\
\hline \multicolumn{4}{|l|}{$\mathrm{RT}, \mathrm{s}$} \\
\hline F1 & 17 (13 to 21$)$ & $16(11$ to 21$)$ & 0.100 \\
\hline $\mathrm{F} 2$ & $17(14$ to 21$)$ & $19(13$ to 22$)$ & \\
\hline F3 & 18 (13 to 22$)$ & $22(15$ to 25$)$ & \\
\hline Intergroup P & 0.568 & 0.016 & \\
\hline \multicolumn{4}{|l|}{$\mathrm{CT}, \mathrm{s}$} \\
\hline F1 & $41(35$ to 45$)$ & $47(40$ to 53$)$ & 0.017 \\
\hline $\mathrm{F} 2$ & $42(35$ to 46$)$ & 52 (40 to 56$)$ & 0.010 \\
\hline F3 & $42(38$ to 48$)$ & $54(38$ to 61$)$ & 0.023 \\
\hline Intergroup $\mathrm{P}$ & 0.688 & 0.275 & \\
\hline
\end{tabular}

B. Venous Response

\begin{tabular}{lllc}
\hline Parameter & Controls $(\boldsymbol{n}=30)$ & Patients $(\boldsymbol{n}=\mathbf{2 4})$ & $\boldsymbol{P}$ \\
\hline Size vein, AU & $147(17)$ & $156(22)$ & 0.061 \\
MD, \% & & & \\
F1 & $6.3(4.2$ to 8.4$)$ & $5.0(3.8$ to 5.6$)$ & 0.168 \\
F2 & $5.7(4.4$ to 7.1$)$ & $5.4(3.9$ to 6.8$)$ & \\
F3 & $6.1(4.7$ to 7.2$)$ & $5.6(5.4$ to 6.5$)$ & \\
Intergroup $P$ & 0.954 & 0.954 & \\
MC, \% & & & \\
F1 & $-1.4(-2.0$ to -0.6$)$ & $-1.5(-2.4$ to -0.5$)$ & 0.841 \\
F2 & $-1.7(-2.2$ to -0.8$)$ & $-1.7(-2.2$ to -0.9$)$ & \\
F3 & $-1.1(-1.8$ to -0.5$)$ & $-1.5(-2.1$ to -0.4$)$ & \\
Intergroup $P$ & 0.367 & 0.368 & \\
DA, \% & & & \\
F1 & $7.7(4.9$ to 9.4$)$ & $6.4(4.7$ to 7.7$)$ & 0.285 \\
F2 & $7.4(5.2$ to 8.8$)$ & $7.1(5.8$ to 8.4$)$ & \\
F3 & $7.2(5.5$ to 8.5$)$ & $7.1(5.9$ to 7.9$)$ & \\
Intergroup $P$ & 0.263 & 0.264 & \\
RT, s & & & \\
F1 & $20(18$ to 22$)$ & $21(14$ to 24$)$ & 0.374 \\
F2 & $19(17$ to 21$)$ & $20(17$ to 24$)$ & \\
F3 & $18(15$ to 21$)$ & $21(17$ to 24$)$ & \\
Intergroup $P$ & 0.142 & 0.896 & \\
\hline
\end{tabular}

$P$ values for arterial response are by ANOVA and for venous response are by ANOVA and Tukey. Data are presented as the mean (IQR). All data are shown as percentage of change compared with the corresponding baseline diameter. F1/F2/F3, flicker 1/flicker 2/flicker 3 (representing the three flicker cycles).

in ophthalmic vessels and in nail-fold capillaries versus systemic and cardiac indices. In the CAD patients, average retinal artery BFR correlated with both circadian HF and LF heart rate variability $(r=0.58, P=0.004 ; r=-0.6, P=0.003$, respec- tively). In the healthy controls, these indices failed to correlate significantly $(r=0.173, P=0.408 ; r=-0.181, P=0.388$, respectively).

\section{Discussion}

We present data on ocular blood vessels and capillary blood flow in CAD, in addition to systemic hemodynamic indices and plasma markers, compared with healthy age- and sex-matched controls. The frequency of men and women in the study does not reflect the normal distribution of the sexes in the healthy population (i.e., 50/50), but is more reflective of CAD which is more prevalent in men. The actual proportions of the sexes reflect those in our cardiology clinics. We did not prospectively favor either sex during the recruitment process. As expected, the patients had adverse arterial stiffness, plasma markers, and heart rate variability compared with the controls. Our new findings are that the patient's CRVs were larger than those of the controls and that a higher proportion of the patients' CRAs showed abnormal spectral wave shape. Furthermore, the CAD patients had OAs (but not veins) that showed abnormal reactivity to flicker light provocation and slower reaction time. Although there was no difference in the velocity of nail-fold capillary blood flow in the patients compared with the controls, the patients had blood vessels that needed considerably longer to recover blood flow after cold provocation. The average retinal artery responses to flickering light correlated with LF and HF heart rate variability, in a positive $(r=$ $0.58)$ and inverse $(r=-0.6)$ manner. That these indices correlate suggests that the abnormalities in our patients' heart rate variability (possibly driven by dysfunctional autonomic nervous system processes) was related to the abnormal responses of ocular arteries. Whether this relationship is pathophysiological, we cannot say.

Pressler et al. ${ }^{48}$ recently compared ocular vessel indices in patients with cardiovascular disease. In their cases with aortic coarctation and high blood pressure, retinal arteriolar vessel diameter (but not venous diameter) was significantly reduced compared with that in controls. No differences in dynamic retinal responses were found, although their analyses were based on averaged responses. Unlike them, we were unable to find any significant correlations between any of our research indices and body mass index (BMI), although all our patients had CAD, did not have high blood pressure, and were older with a higher BMI than those of Pressler et al. Traditionally, large-artery assessments have been used for the assessment of vascular function. However, many of these tests are invasive, have poor reproducibility, and are not informative regarding the microcirculation. Instead, the eyes and nail-folds are convenient locations for noninvasive assessment of structural and functional assessment of the microcirculation in several cardiovascular disorders. ${ }^{9,17-41}$ The eye itself is very vulnerable to minor changes in perfusion, leading to structural and functional abnormalities. ${ }^{49}$ It is therefore a suitable site to investigate early changes that may predict large vessel disease. ${ }^{18}$

Our CAD patients were free of DM and HT and were all well motivated to attend a rehabilitation course. However, most patients received vasculoactive treatments that could have masked structural vessel changes. In common with other studies, our patient group showed increased BMI and retinal venous diameter. ${ }^{50}$ Functional and structural changes are not necessarily concurrent, although vasculature that appears to be structurally normal can have functional impairment. ${ }^{51}$. Furthermore, static retinal diameter measurements can show variations of up to $34 \%$ which arise due to observer variability and the particular stage of cardiac cycle in which they were taken. ${ }^{52}$ When ocular vessel diameters are measured continu- 
ously, these limitations can be addressed. In addition our data show that although the structure of retinal arteries seemed to be comparable between the groups, the functional aspects are already altered in the CAD group. The retinal arterioles of the CAD patients showed a significant decrease in the reaction pattern, which manifested as diminished dilatory response with gradually longer reaction times with each flicker cycle. The time needed to reach MC was also increased in the CAD patients compared with the controls. The arteriosclerotic vessel wall changes causing an overall stiffening alongside an imbalance of local regulatory factors such as endothelin 1 and nitric oxide are the factors most likely to contribute to an increase in reaction and constriction time of retinal arterioles in CAD patients. These results (in combination with their imbalance in ANS function showing sympathetic overdrive and increased levels of plasma markers) show systemic endothelial dysfunction, not only at the large artery level but simultaneously at the microcirculatory vascular beds in different parts of the body. The absence of any correlation between nail-fold and ocular circulation indices in health or in CAD supports the view that both vascular systems operate independently. Indeed, the fact that none of our other research or routine indices correlated with the abnormal nail-fold capillary responses in CAD implies the presence of an alternative pathophysiological mechanism.

Our data are limited by the possible effects of various systemic medications being taken by the patients and that we recruited from a well-motivated group that may contribute to a better than expected endothelial function compared with poorly motivated patients on different medications. However, similar to other studies ${ }^{27}$ we attempted to minimize the effect of medication by discontinuing it for 24 hours, Nevertheless, our patients had documented CAD, and we have demonstrated their endothelial, blood pressure, and heart rate abnormalities.

We conclude that assessment of the retina may be a useful tool in diagnosis of cardiovascular disease, as well as in monitoring disease progression and the efficacy of treatment.

The comparison of the presented results may be limited, since the RVA data analysis was not standardized across research groups.

\section{References}

1. Seddon MD, Chowienczyk PJ, Brett SE, Casadei B, Shah AM. Neuronal nitric oxide synthase regulates basal microvascular tone in humans in vivo. Circulation. 2008;117:1991-1996.

2. Wehrwein EA, Joyner MJ, Hart EC, Wallin BG, Karlsson T, Charkoudian N. Blood pressure regulation in humans: calculation of an "error signal" in control of sympathetic nerve activity. Hypertension. 2010;55:264-269.

3. Sica DA. What are the influences of salt, potassium, the sympathetic nervous system, and the renin-angiotensin system on the circadian variation in blood pressure? Blood Press Monit. 1999; 4(suppl 2):S9-S16.

4. Valentini M, Parati G. Variables influencing heart rate. Prog Cardiovasc Dis. 2009;52:11-19.

5. Blann AD. How a damaged blood vessel wall contributes to thrombosis and hypertension. Path Haemostas Thromb. 2004:33;445448.

6. Rechciński T, Trzos E, Wierzbowska-Drabik K, Krzemińska-Pakuła M, Kurpesa M. Melatonin for nondippers with coronary artery disease: assessment of blood pressure profile and heart rate variability. Hypertens Res. 2010;33:56-61.

7. Evrengul H, Tanriverdi H, Kose S, Amasyali B, Kilic A, Celik T, Turhan $\mathrm{H}$. The relationship between heart rate recovery and heart rate variability in coronary artery disease. Ann Noninvasive Electrocardiol. 2006;11:154-162.

8. Fukuda D, Yoshiyama M, Shimada K, et al. Relation between aortic stiffness and coronary flow reserve in patients with coronary artery disease. Heart. 2006;92:759-762.
9. Stubiger N, Erb C, Rohrbach JM, and Thiel HJ. Pericorneal vascular changes as an expression of systemic vascular processes (in German). Klin Monatsbl Augenheilkd. 1997;210:69-73.

10. Agabiti-Rosei E. From macro- to microcirculation: benefits in hypertension and diabetes. J Hypertens Suppl. 2008;26:S15-S19.

11. Romero-Aroca P, Mendez-Marin I, Baquet-Bernaldiz M, FernéndezBallart J, Santos Blanco E. Review of the relationship between renal and retinal microangiopathy in diabetes mellitus patients. Curr Diabetes Rev. 2010;6:88-101.

12. Hughes AD. The clinical assessment of retinal microvascular structure and therapeutic implications. Curr Treat Options Cardiovasc Med. 2007;9:236-241.

13. Debbabi H, Bonnin P, Ducluzeau PH, Lefthériotis G, Levy BI. Noninvasive assessment of endothelial function in the skin microcirculation. Am J Hypertens. 2010;23:541-546.

14. Hammer M, Vilser W, Riemer T, et al. Diabetic patients with retinopathy show increased retinal venous oxygen saturation. Graefes Arch Clin Exp Ophthalmol. 2009;247:1025-1030.

15. Czernichow S, Greenfield JR, Galan P, et al. Microvascular dysfunction in healthy insulin-sensitive overweight individuals. $J$ Hypertens. 2010;28:325-332.

16. Nguyen TT, Kawasaki R, Wang JJ, et al. Flicker light-induced retinal vasodilation in diabetes and diabetic retinopathy. Diabetes Care. 2009;32:2075-2080.

17. Senn B, Orgul S, Keller U, et al. Retrobulbar and peripheral capillary blood flow in hypercholesterolemic subjects: review of the methods used. Am J Ophthalmol. 1999;128:310-316.

18. Liew G, Wang JJ, Mitchell P, Wong TY. Retinal vascular imaging: a new tool in microvascular disease research. Circ Cardiovasc Imaging. 2008;1:156-161.

19. Wong TY, Klein R, Klein BE, Tielsch JM, Hubbard L, Nieto FJ. Retinal microvascular abnormalities and their relationship with hypertension, cardiovascular disease, and mortality. Surv Ophthalmol. 2001;46:59-80.

20. Fujioka S, Karashima K, Nishikawa N, Saito Y. Ocular circulation in diabetic patients with coronary artery disease. Graefes Arch Clin Exp Ophthalmol. 2006;244:163-169.

21. Gilani M, Kaiser DR, Bratteli CW, et al. Role of nitric oxide deficiency and its detection as a risk factor in prehypertension. $\mathrm{J} \mathrm{Am}$ Soc Hypertens. 2007;1:45-55.

22. Dolan E, Thijs L, Li Y, Atkins N, et al. Ambulatory arterial stiffness index as a predictor of cardiovascular mortality in the Dublin outcome study. Hypertension. 2006;47:365-370.

23. Duprez DA, Cohn JN. Arterial stiffness as a risk factor for coronary atherosclerosis. Curr Atheroscler Rep. 2007;9:139-144.

24. Blann AD, Taberner DA. A reliable marker of endothelial cell dysfunction: does it exist? Br J Haematol. 1995;90:244-248.

25. Patton N, Aslam T, Macgillivray T, Pattie A, Deary IJ, Dhillon B. Retinal vascular image analysis as a potential screening tool for cerebrovascular disease: a rationale based on homology between cerebral and retinal microvasculatures. J Anat. 2005;206:319-348.

26. Patton N, Aslam TM, Macgillivray T, et al. Retinal image analysis: concepts, applications and potential. Prog Retin Eye Res. 2006; 25:99-127.

27. Lind L. Arterial compliance influences the measurement of flowmediated vasodilation, but not acetylcholine-mediated forearm blood flow: the prospective investigation of the vasculature in Uppsala seniors (pivus) study. Atherosclerosis. 2007;190:212-215.

28. Dolan E, Li Y, Thijs L, et al. Ambulatory arterial stiffness index: rationale and methodology. Blood Press Monit. 2006;11:103-105.

29. Li Y, Dolan E, Wang JG, et al. Ambulatory arterial stiffness index: determinants and outcome. Blood Press Monit. 2006;1:107-110.

30. Bracewell RN. Fourier Analysis and Imaging. Stuttgart, Germany: Springer; 2003.

31. Sleight P, La Rovere MT, Mortara A, et al. Physiology and pathophysiology of heart rate and blood pressure variability in humans: is power spectral analysis largely an index of baroreflex gain? Clin Sci. 1995;88:103-109.

32. Pagani M, Lombardi F, Guzzetti S, et al. Power spectral analysis of heart rate and arterial pressure variabilities as a marker of sympatho-vagal interaction in man and conscious dog. Circ Res. 1986; 59:178-193. 
33. Haerten R, Kim J. Procedures in color Doppler ultrasoundcomparison of methods (in German). Ultraschall Med. 1993;14: 225-230.

34. Polak K, Dorner G, Kiss B, et al. Evaluation of the Zeiss retinal vessel analyser. Br J Ophthalmol. 2000;84:1285-1290.

35. Hubbard LD, Brothers RJ, King WN, et al. Methods for evaluation of retinal microvascular abnormalities associated with hypertension/sclerosis in the atherosclerosis risk in communities study. Opbthalmology. 1999;106:2269-2280.

36. Parr JC, Spears GF. General caliber of the retinal arteries expressed as the equivalent width of the central retinal artery. $A m J O p h$ thalmol. 1974;77:472-477.

37. Blum M, Bachmann K, Wintzer D, Riemer T, Vilser W, Strobel J Noninvasive measurement of the Bayliss effect in retinal autoreg ulation. Graefes Arch Clin Exp Opbthalmol. 1999;237:296-300.

38. Seifertl BU, Vilser W. Retinal Vessel Analyzer (RVA): design and function. Biomed Tech (Berl). 2002;47(suppl 1):678-681.

39. Garhofer G, Bek T, Boehm AG, et al. Use of the retinal vessel analyzer in ocular blood flow research. Acta Opbthalmol. 2010; 88:717-722

40. Heitmar R, Blann AD, Cubbidge RP, Lip GYH, Gherghel D. Continuous retinal vessel diameter measurements: the future of retinal vessel assessment? Invest Ophthalmol Vis Sci. 2010;51:58335839.

41. Portig I, Maisch B. Noninvasive methods in the diagnosis of macroand microangiopathy of peripheral and carotid arteries (in German). Herz. 2004;29:17-25.

42. Pries AR, Habazettl H, Ambrosio G, et al. A review of methods for assessment of coronary microvascular disease in both clinical and experimental settings. Cardiovasc Res. 2008;80:165-174.
43. Saner H, Wuerbel F, Mahler F, Flammer J, Gasser P. Microvasculatory evaluation of vasospastic syndromes. Adv Exp Med Biol. 1987;220:215-218

44. Gasser P, Prünte-Glowazki A. Ocular vasospasm, 2: Diagnosis of vasospastic syndrome using nailfold capillary videomicroscopy (in German). Klin Monbl Augenbeilkd. 1991;198:412-413.

45. Gasser P, Dubler B. Z Development of instrumental and technical measurement aspects for clinical capillary microscopy. Rbeumatology. 1996;55:260-266.

46. Stuecker M, Baier V, Reuther T, Hoffmann K, Kellam K, Altmeyer P. Capillary blood cell velocity in human skin capillaries located perpendicularly to the skin surface: measured by a new laser Doppler anemometer. Skin Res Technol. 2004;10:174-177.

47. Machin D, Campbell M. Statistical Tables for the Design of Clinical Trials. Oxford, UK: Blackwell Scientific; 1987.

48. Pressler A, Esefeld K, Scherr J, et al. Structural alterations of retinal arterioles in adults late after repair of aortic isthmic coarctation. Am J Cardiol. 2010;105:740-744.

49. Flammer J, Mozaffarieh M. Autoregulation, a balancing act between supply and demand. Can J Opbthalmol. 2008;43:317-321.

50. Kawasaki R, Cheung N, Wang JJ, et al. Retinal vessel diameters and risk of hypertension: the Multiethnic Study of Atherosclerosis. J Hypertens. 2009;27:2386-2393.

51. Green DJ. Exercise training as vascular medicine: direct impacts on the vasculature in humans. Exerc Sport Sci Rev. 2009;37:196-202.

52. Newsom RS, Sullivan PM, Rassam SM, Jagoe R, Kohner EM. Retinal vessel measurement: comparison between observer and computer driven methods. Graefes Arch Clin Exp Opbthalmol. 1992;230: 221-225. 